\title{
A rare case of nonenhancing primary central nervous system lymphoma mimic multiple sclerosis
}

\author{
Hai Chen, MD, Huiqing Dong, MD.
}

\begin{abstract}
تفيد التقارير أن سرطان الغدد الليمفاوية في الجهاز العصبي المركزي

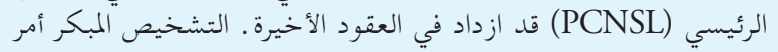

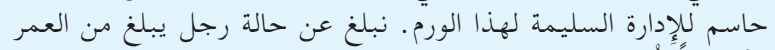

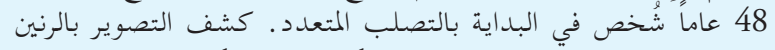

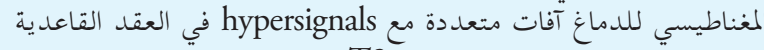

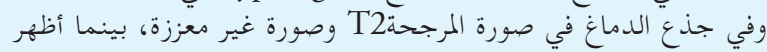

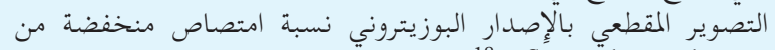
مأ

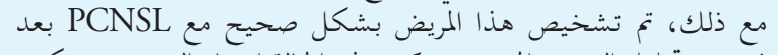

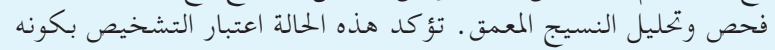

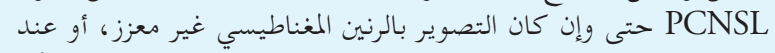

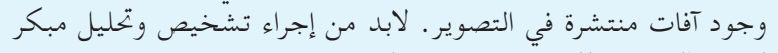

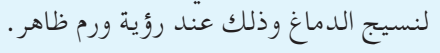

Primary central nervous system lymphoma (PCNSL) is reported to have increased in the last decades. Early diagnosis is crucial for proper management of this tumor. We report a case of a 48-year-old man who was initially diagnosed with multiple sclerosis. Magnetic resonance imaging of the brain revealed multiple lesions with hypersignals in the bilateral basal ganglia and brain stem in T2-weighted image and nonenhancement, while positron emission tomography showed a low uptake of ${ }^{18} \mathrm{~F}$-fluorodeoxyglucose in the affected brain, indicative of demyelination. However, this individual was correctly diagnosed with PCNSL after biopsy and further histological analysis. Primary central nervous system lymphoma must be considered even when nonenhancing, diffuse lesions are seen on MRI. A visible tumor on imaging is essential to ensure an early brain biopsy and histological diagnosis.

Neurosciences 2015; Vol. 20 (4): 380-384 doi: 10.17712/nsj.2015.4.20150125

From the Department of Neurology, Xuanwu Hospital of Capital Medical University, Beijing, People's Republic of China.

Received 10th February 2015. Accepted 29th June 2015.

Address correspondence and reprint request to: Dr. Huiqing Dong, Department of Neurology, Xuanwu Hospital of Capital Medical University, Beijing 100053, People's Republic of China. Fax.+86(10)83167306.E-mail: ch-123456-hai@163.com
Drimary central nervous system lymphoma (PCNSL) 1 accounts for $3.3 \%$ of all brain tumors. ${ }^{1}$ Primary central nervous system lymphoma in immunocompetent patients is non-Hodgkin lymphomas of germinal B-cell origin in the vast majority of cases, which arise from the brain, spinal cord, cerebrospinal fluid (CSF), or eyes in the absence of systemic disease. ${ }^{1,2}$ Because of its ambiguous clinical manifestation, neuroradiographic, and CSF cytology, PCNSL can be easily misdiagnosed. Our objective in presenting this particular case is to highlight that PCNSL should be considered even when non-enhancing, diffuse lesions are seen on MRI.

Case Report. A 48-year-old Chinese male with a history of hypersomnia for 4 months and a progressive dementia for 3 months was admitted to our hospital. This patient claimed that he had been in excellent health until 4 months before admission and experienced hypersomnia (10-12 hours/day), but with normal working and eating patterns. He reported a decline in hearing during the 2 months prior to his admission and he had got lost once during this period. However, he denied the history of fever and persistent weight loss, as well as the abnormal family's medical histories. On referral to that hospital, he was diagnosed as demyelination and treated with methylprednisolone (500 mg intravenous injection per day, for 3 days, but without any medications). After steroid treatment, his symptoms progressively worsened. He developed aphasia with the severely impaired memory and could not make out objects. He completely lost his hearing, and developed urinary and fecal incontinence 4 weeks after hospital admission.

On neurological examination, he was found to be apathetic with dementia, motor and sensory aphasia, as well as an inability to communicate. Fundoscopic

Disclosure. Authors have no conflict of interests, and the work was not supported or funded by any drug company. 
examination of the eyes revealed the normal retinas and optic discs except the hearing loss of double ears. Muscular tension was normal and muscle strength was $5 / 5$ in all extremities. Tendon reflexes were normal, bilateral Babinski signs were negative, and both Kernig's and Brudzinski's signs were negative. The patient was uncooperative during examinations of coordination and sensation. Markers of autoimmune diseases, such as antinuclear antibodies, extractable nuclear antigen, and antineutrophil cytoplasmic antibodies, were all negative.
Serum lactate dehydrogenase was normal (206IU/L). Analyses of viral antibodies, syphilis serology, and human immunodeficiency virus antibodies was negative. Lumbar puncture showed a normal intracranial pressure ( $80 \mathrm{~mm} \mathrm{H}_{2} \mathrm{O}$ ). In the CSF findings, tumor cells, white, or red blood cells were not observed. The protein level was slightly elevated $(59 \mathrm{mg} / \mathrm{dL})$, however, chloride $(118 \mathrm{mmol} / \mathrm{L})$ and glucose $(58 \mathrm{mg} / \mathrm{dL})$ were normal. Magnetic resonance imaging of the brain revealed multiple lesions with hypersignals in the bilateral basal
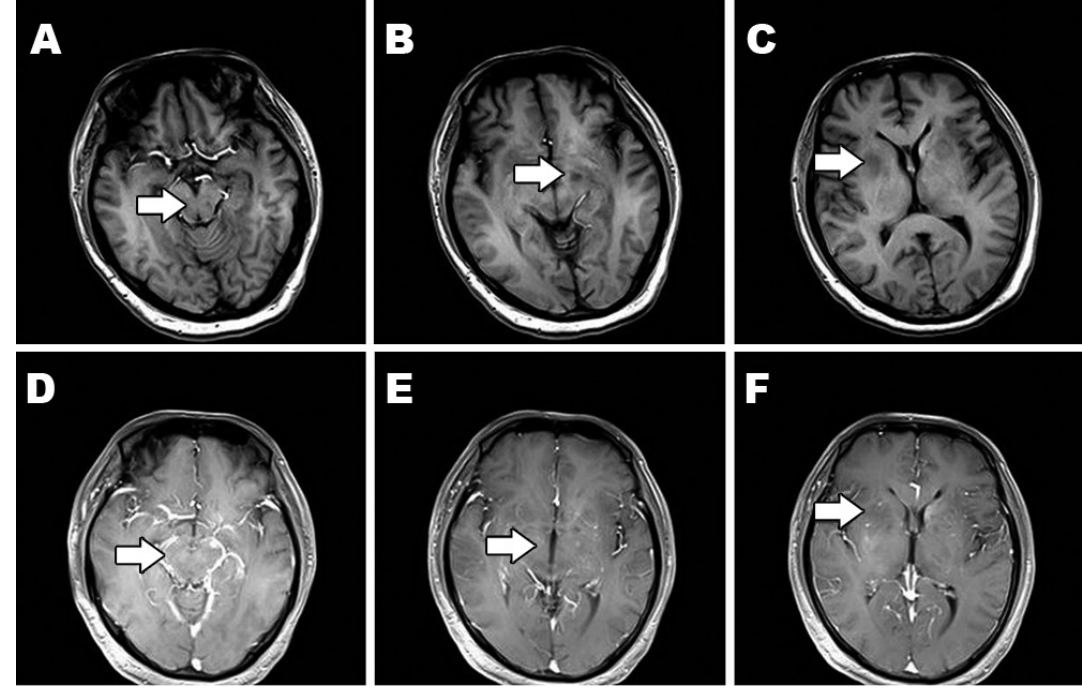

Figure 1 - Magnetic resonance imaging of the brain showing: A-C) multiple lesions with hyposignals in the bilateral basal ganglia and brain stem in T1-weighted image (arrow). D-F) these lesions are non-enhancement in contrast image.
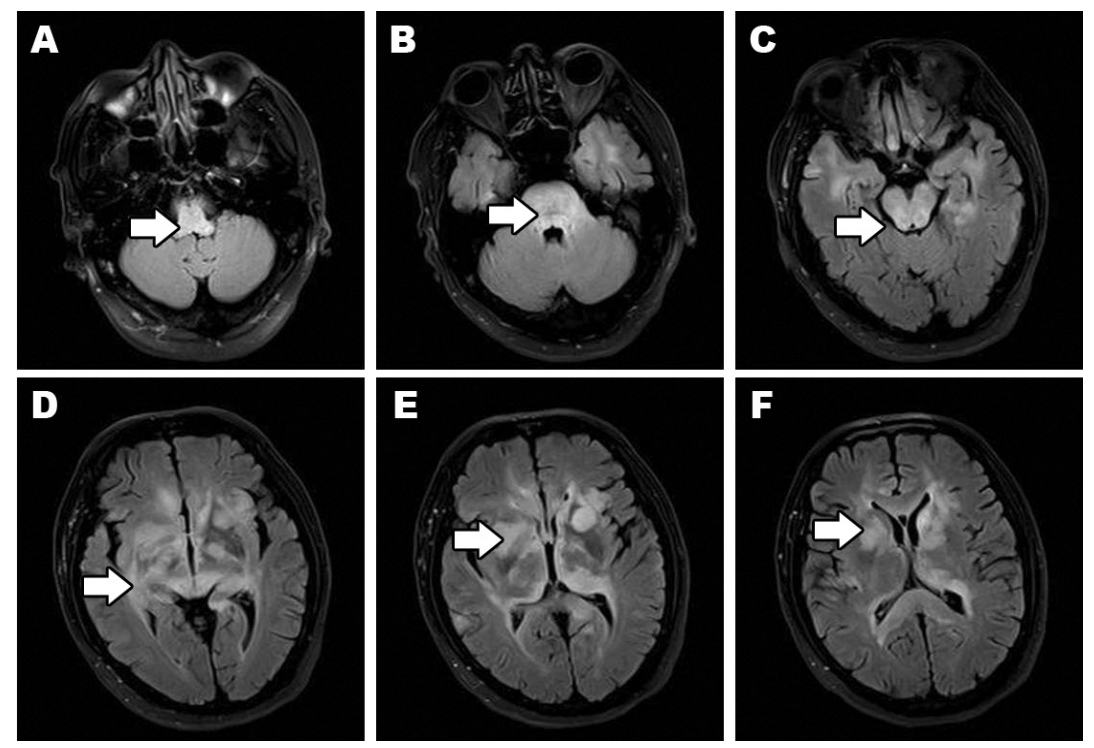

Figure 2 - Serial MR scans showing: A-F) more lesions with hypersignals in fluid attenuated inversion recovery, A) the lesions involved the bilateral medulla oblongata, B) pons, C) midbrain, D) thalamus, E) basal ganglia, D-F) corpus callosum and temporal lobe involving almost the entire brain (arrows). 
ganglia and brain stem in T2-weighted image (T2WI) and non-enhancement (Figure 1).

The initial diagnosis was considered as idiopathic inflammatory demyelinating disease with methylprednisolone treatment. After one-month treatment with no signs of improvement, serial magnetic resonance (MR) scans revealed more lesions with hypersignals using fluid attenuated inversion recovery (Figure 2). The lesions were detected in the bilateral medulla oblongata, pons, midbrain, thalamus, basal ganglia, corpus callosum, and temporal lobe, which were located in almost the entire brain. A gadolinium-based contrast image showed non-enhancement and positron emission tomography (PET) showed a low ${ }^{18} \mathrm{~F}$-Fludeoxyglucose $\left({ }^{18} \mathrm{~F}-\mathrm{FDG}\right)$ uptake in the affected brain, indicating demyelination (Figure 3). Stereotactic biopsy of the right temporal
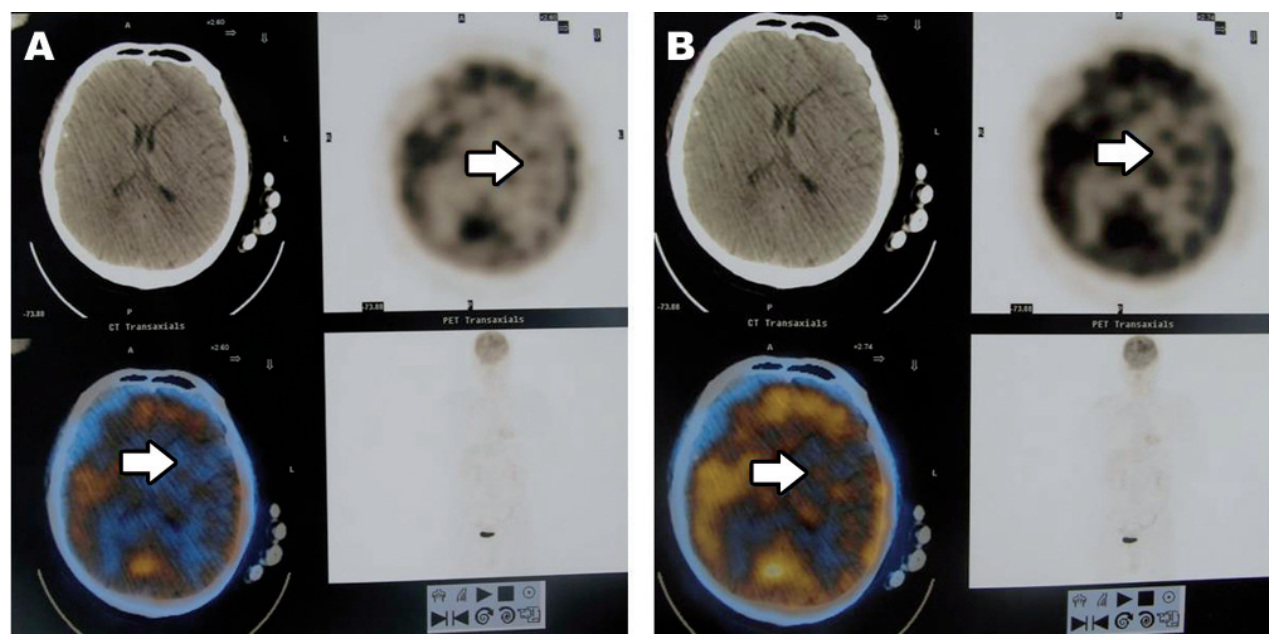

Figure 3 - Positron emission tomography of the brain showing A+B) a low ${ }^{18} \mathrm{~F}-\mathrm{FDG}$ uptake in the affected brain, indicating demyelination.
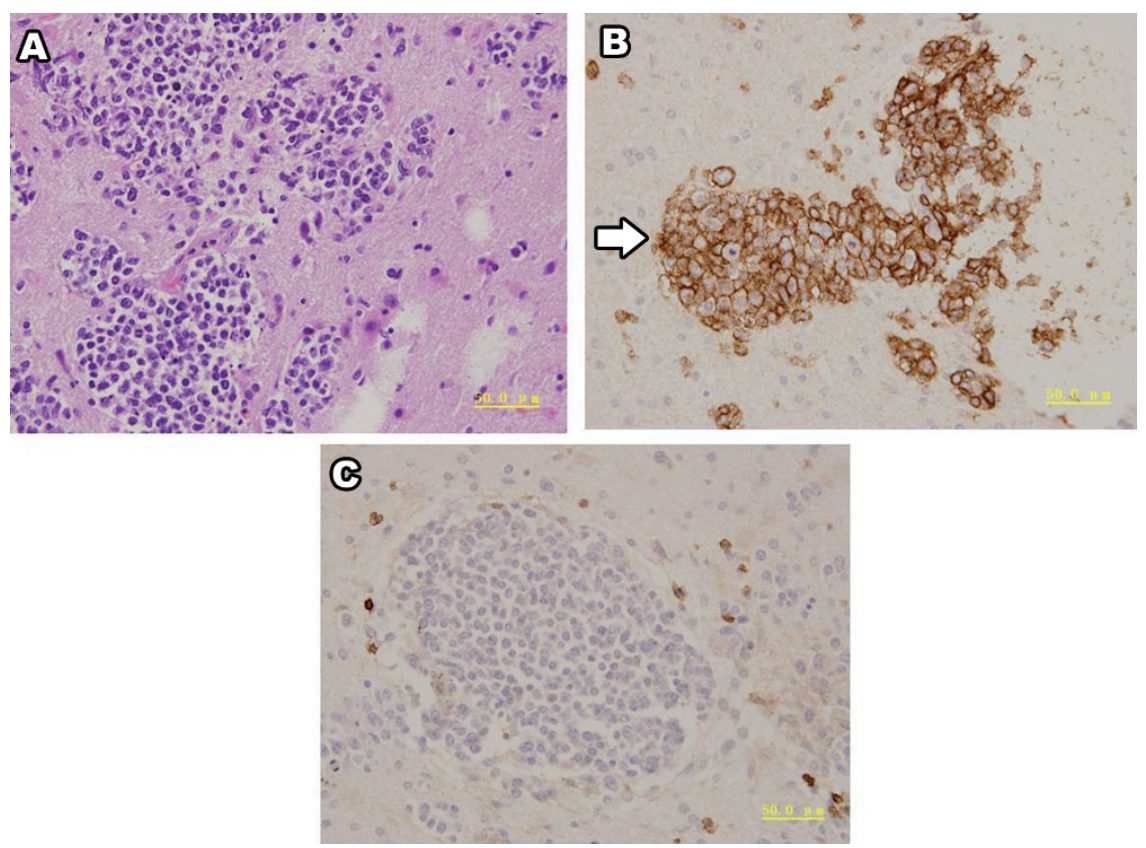

Figure 4 - Pathology of the brain showing: A) Hematoxylin-eosin (HE) staining, B) immunohistochemistrystaining of the brain mass biopsy with the B-cell marker CD20 (+) (arrow), and C) immunohistochemistry staining of the brain mass biopsy with the T-cell marker CD45RO (-). 
lobe was performed and immunohistochemical analysis showed that the brain tissue was infiltrated with multiple foci of heterotypic cells withCD20 (+) and CD45RO (-) (Figure 4), suggestive of non-Hodgkin malignant B-cell lymphoma. After obtaining these results, the patient was given radio-chemotherapy. Whole-brain radiation therapy (WBRT) started with 45 Gy and no boost. Methotrexate was used at a high dose $\left(4 \mathrm{~g} / \mathrm{m}^{2}\right)$ in chemotherapy cycles with a 3 weekly interval. After the sixth course of methotrexatecycle, external radiotherapy was applied to WBRT. Acute chemotherapy-related toxicities such as nausea and vomiting were observed and toxicity due to radiotherapy was only seen in dermal and mucosal tissue. All these side effects disappeared after symptomatic treatment. Six months after admission, he recovered and could hear some words with enough speech for simple communication, and he was also able to remember things to a certain extent. Magnetic resonance imaging of the brain showed that the lesions were decreased. Unfortunately, 25 months after first admission, he died of pulmonary infection. The family member gave consent to publish this case report.

Discussion. Magnetic resonance imaging is considered to be the most useful imaging modality in the diagnosis of PCNSL as most lesions show a marked enhancement effect with contrast material. However, contrast-enhanced MRI do not always clearly differentiate PCNSL from other neoplasms (such as, metastases, malignant gliomas), or nonneoplastic diseases (such as, multiple sclerosis [MS], stroke). ${ }^{3}$ Positron emission tomography studies with FDG and ${ }^{11} \mathrm{C}$-methionine (MET) usually show strong accumulation of tracers in PCNSL and have been reported to be useful in distinguishing PCNSL from other lesions. ${ }^{4}$

Nonenhancing PCNSL has been thought to be very rare. ${ }^{5}$ Terae and Ogata ${ }^{5}$ reported the first case of completely nonenhancing PCNSL on MRI (before the patient had received any treatment, including steroids). They suggested that lesions with relatively sparse infiltration of tumor cells did not enhance, presumably because the blood-brain barrier was intact.

Most PCNSLlesions affect the frontal lobe, the corpus callosum, and the basal ganglia. ${ }^{6}$ On pre-contrast MRI, PCNSL is known to appear as hypo- or iso-intense on a T1-weighted image (T1WI), and hyper- or iso-intense on the T2WI. In comparison, the enhancement after injection of gadopentetate dimeglumine is known to be variable $^{7}$ and can even occur to a different degree within the same patient in multifocal disease. Single or multiple periventricular lesions with gadolinium enhancement are typical findings in immunocompetent patients, whereas ring enhancing lesions are only observed in immunocompromised patients. ${ }^{8}$ Zhang et $\mathrm{al}^{6}$ reported an 'open ring' and a 'notch sign' as enhancement patterns. Calcifications, cyst formations, and hemorrhage are rare findings, which might be indicative of other diseases. ${ }^{6}$ Kuker et $\mathrm{al}^{9}$ evaluated pre-treatment MRI scans in a cohort of 100 immunocompetent patients to delineate the characteristic presenting imaging features of PCNSL. In one patient, contrast enhancement was entirely absent, in spite of biopsy-proven B-cell lymphoma. The non-enhancement frequency in PCNSL seems to be less than $1 \%$. The lesions of this patient are located around the ventricular system and this is typical characteristics of PCNSL. Some of the lesions in this patient are located in the gray matter, and MS is the disease that invades the white matter. Therefore, expert neurologists and radiologists could diagnose this patient as suffering from PCNSL not MS. Moreover, treatment with steroid often blurs the lesions in contrast-enhanced MRI.

Primary central nervous system lymphoma demonstrated high FDG uptake and can be diagnosed by FDG-PET with high sensitivity. ${ }^{10}$ In contrast, the patient in this study showed low FDG uptake. Kawai et $\mathrm{al}^{4}$ conducted a study to examine the usefulness of PET examinations with FDG and MET in the diagnosis of PCNSL patients with atypical MRI, by comparing their PET results with those from PCNSL patients with typical MRI. Positron emission tomography with FDG and MET can measure the glucose and amino acid metabolism in lesions and may provide useful information to diagnose PCNSL in patients with more subtle MRI results. However, visual analysis of FDG and MET uptake in atypical PCNSL was not useful to find lesions in the brain. Furthermore, semi-quantitative and quantitative uptake values obtained from lesions with atypical MRI results were not useful for differentiating PCNSL from other tumorous and non-tumorous diseases. The data we obtained from PET were consistent with previous studies. ${ }^{10} \mathrm{We}$ should consider cumulative dose of corticosteroid, because accumulation of corticosteroid before a PET scan could influenced FDG uptake. ${ }^{11}$

In conclusion, PCNSL must be considered even when nonenhancing, diffuse lesions are seen on MRI. Primary central nervous system lymphoma is hard to diagnose since PCNSL mimics MS both clinically and radiologically. In some cases, PET should be carried out more than once, and brain biopsy could be performed when PCNSL is strongly suspected. 
Acknowledgments. The authors would like to thank Dr. Yunyun Duan (Radiologist) for revising the MRIs, and Dr. Guocai Tang (Pathologist) for the pathology images.

\section{References}

1. Gerstner ER, Batchelor TT. Primary central nervous system lymphoma. Arch Neurol 2010; 67: 291-297.

2. Zhao D, Qian L, Shen J, Liu X, Mei K, Cen J, et al. Combined treatment of rituximab, idarubicin, dexamethasone, cytarabine, methotrexate with radiotherapy for primary central nervous system lymphoma. J Cell Mol Med 2014; 18: 1081-1086.

3. Haldorsen IS, Krakenes J, Krossnes BK, Mella O, Espeland A. $\mathrm{CT}$ and MR imaging features of primary central nervous system lymphoma in Norway, 1989-2003. AJNR Am J Neuroradiol 2009; 30: 744-751.

4. Kawai N, Okubo S, Miyake K, Maeda Y, Yamamoto Y, Nishiyama Y, et al. Use of PET in the diagnosis of primary CNS lymphoma in patients with atypical MR findings. Ann Nucl Med 2010; 24: 335-343.

5. Terae S, Ogata A. Nonenhancing primary central nervous system lymphoma. Neuroradiology 1996; 38: 34-37.
6. Zhang D, Hu LB, Henning TD, Ravarani EM, Zou LG, Feng XY, et al. MRI findings of primary CNS lymphoma in 26 immunocompetent patients. Korean J Radiol 2010; 11: 269-277.

7. Johnson BA, Fram EK, Johnson PC, Jacobowitz R. The variable MR appearance of primary lymphoma of the central nervous system: comparison with histopathologic features. AJNR Am J Neuroradiol 1997; 18: 563-572.

8. Soussain C, Hoang-Xuan K. Primary central nervous system lymphoma: an update. Curr Opin Oncol 2009; 21: 550-558.

9. Kuker W, Nagele T, Korfel A, Heckl S, Thiel E, Bamberg M, et al. Primary central nervous system lymphomas (PCNSL): MRI features at presentation in 100 patients. J Neurooncol 2005; 72 : 169-177.

10. Palmedo H, Urbach H, Bender H, Schlegel U, Schmidt-Wolf IG, Matthies A, et al. FDG-PET in immunocompetent patients with primary central nervous system lymphoma: correlation with MRI and clinical follow-up. Eur J Nucl Med Mol Imaging 2006; 33: 164-168.

11. Yamaguchi S, Hirata K, Kobayashi H, Shiga T, Manabe O, Kobayashi K, et al. The diagnostic role of (18)F-FDG PET for primary central nervous system lymphoma. Ann Nucl Med 2014; 28: 603-609.

REFERENCES
* References should be primary source and numbered in the order in which they appear in the
text. At the end of the article the full list of references should follow the Vancouver style.
* Unpublished data and personal communications should be cited only in the
text, not as a formal reference.
* The author is responsible for the accuracy and completeness of references
and for their correct textual citation.
* When a citation is referred to in the text by name, the accompanying
reference must be from the original source.
* Upon acceptance of a paper all authors must be able to provide the full paper
for each reference cited upon request at any time up to publication.
* Only 1-2 up to date references should be used for each particular point in
the text.

